\title{
Antifungal Susceptibility Profile of Candida and its Comparison in Various Candida Species
}

\author{
M.V. Natasha ${ }^{1^{*}}$ and Y. Raksha ${ }^{2}$ \\ ${ }^{1}$ Department of Microbiology, Gulbarga Institute of Medical Sciences Veeresh Nagar, \\ Gulbarga-585105, India \\ ${ }^{2}$ Department of Microbiology, ESIC-MC-PGIMSR, Rajajinagar, Bengaluru-560010, India \\ *Corresponding author:
}

\section{A B S T R A C T}

\section{Keywords}

Candida albicans,

Voriconazole, population

\section{Article Info}

Accepted:

24 May 2019

Available Online:

10 June 2019
Candida spp. can cause a wide variety of infections in immunosuppressed patients of long term broad - spectrum antibiotics, steroids or other immunosuppressive agents, diabetes mellitus, AIDS, Malignancy, Neutropenia. Although the majority of infections are caused by Candida albicans, Non-C. albicans Candida (NCAC) species are emerging as important pathogen in humans. Our study included 130 clinical isolates which showed gram positive budding yeasts cells with or without pseudohyphae. $\mathrm{KOH}$ mount, germ tube, Carbohydrate assimilation test and fermentation test and antifungal susceptibility by disc diffusion method was done.65\% of the total isolates were sensitive to Fluconazole and $82 \%$ of the total isolates were sensitive to Voriconazole

\section{Introduction}

With the increase in the population of patients with immunodeficiency or undergoing immunosuppressive therapy, mycoses have become a growing problem in modern medical care. In addition, the diagnosis of these diseases can be problematic, drug resistance is of great concern, and fewer drugs are available compared to bacterial or viral diseases $(1,2,3)$.

Candida is the most prevalent fungal pathogen in humans. Candida is a part of the normal flora in healthy individuals, and is usually confined to the skin and mucosal surfaces of the oral cavity, gastrointestinal and urogenital tracts, and vagina. Although the majority of infections are caused by Candida albicans, Non-C. albicans Candida (NCAC) species are emerging as important pathogen in humans. Candida spp. is also one of the most frequent pathogens isolated in bloodstream infections, associated with significant morbidity and mortality in hospitalized patients (4-6). Fluconazole is an azole group of antifungal agent, been widely used for both treatment and prophylaxis of fungal infections. Due to its limited spectrum of antifungal activity and resistance noticed in 
immunocompromised hosts, second generation triazoles like voriconazole have been developed. Possibility of innate or acquired resistance of Candida spp to antimycotic drugs has emphasized invitro antimycotic susceptibility testing.

Extensive use of antimycotic drugs led to change in prevalence of Candida spp with a decrease in proportion of $C$. albicans and increase in proportion of non albicans such as C glabrata, $C$ krusei.

The present study included 130 clinical isolates which showed gram positive budding yeasts cells with or without pseudohyphae. Our study aimed to determine the in vitro susceptibilities of various Candida species isolates to fluconazole and Voriconazole and to compare both.

\section{Materials and Methods}

A total of 130 Candida species isolated from clinical samples like urine, pus, sputum, blood, oral swabs, vaginal swabs, endotracheal aspirates, endotracheal tips, were included in this study. The study was conducted from November 2012 to April 2014 in the department of Microbiology, ESIC MC PGI MSR Hospital Bangalore. Data of patients regarding the use of long term broad - spectrum antibiotics, steroids or other immunosuppressive agents, diabetes mellitus, AIDS, Malignancy, Neutropenia were collected at enrolment.

Examination of specimen was done by Gram staining, $10 \%$ and $40 \% \mathrm{KOH}$ mount, Cultural characteristics were noted on SDA with chloramphenicol, Germ tube test, chlamydospore formation on cornmeal agar with $1 \%$ tween 80 (by Dalmau technique) speciation was done by Carbohydrate assimilation test by Auxanographic plate method (Haley and standard modification) using Yeast nitrogen base and $20 \%$ sugars. Sugar fermentation test with $6 \%$ sugars was done for speciation.

Antifungal susceptibility test was done by Kirby- Bauer's disc diffusion method on glucose methylene blue Mueller Hinton agar (GM-MH) using commercially available antifungal discs [Hi Media, Mumbai, India] fluconazole $(25 \mu \mathrm{g})$, Voriconazole $1 \mu \mathrm{g}$.(Fig $3)$. Zone diameters were interpreted as per the approved NCCLS (M44-A) guidelines. Antifungal susceptibility test: Antifungal susceptibility test was done by the disc diffusion method on glucose methylene blue Mueller Hinton agar (GM-MH). GM-MH was prepared by addition of $2 \%$ glucose and 0.5 $\mu \mathrm{g} / \mathrm{ml}$ of methylene blue to Mueller Hinton agar. The agar medium should have a $\mathrm{pH}$ of 7.2-7.4 at room temperature after gelling.

The inoculum was prepared by picking five distinct colonies of approximately $1 \mathrm{~mm}$ from 24 hours old cultures grown on Sabouraud's dextrose agar (SDA). Colonies were suspended in $5 \mathrm{ml}$ of sterile $0.85 \%$ saline. This suspension was adjused to turbidity of $0.5 \mathrm{Mc}$ Farlands yielding $1 \times 10^{6}-5 \times 10^{6}$ cells $/ \mathrm{ml}$

\section{Preparation of GM-MH Agar (appendix)}

Inoculation of Test plates-Within 15 minutes of inoculum preparation, the swab dipped in suspension was streaked on the entire surface of GM-MH agar. The antifungal discs were placed $24 \mathrm{~mm}$ apart from each other. The plates were then incubated at $37^{\circ} \mathrm{C}$ for 24 hours.

If insufficient growth was observed after 24 hours, the plates were read after 48 hours. Zone diameters were interpreted as per the approved NCCLS (M44-A) guidelines. The quality control test was performed by using $C$. parapsilosis (ATCC 22019), and C. albicans (ATCC 90028). 
Interpretation of Disk diffusion test resultsZone of inhibition of uniform circular semiconfluent growth is considered. The zone diameter to the nearest whole $\mathrm{mm}$ at the point at which there is prominent reduction in growth is measured.

\section{Results and Discussion}

The chart shows that $65 \%$ of the isolates were sensitive to Fluconazole and $35 \%$ resistant. $82 \%$ were sensitive and $18 \%$ resistant to Voriconazole.

The chart shows that $100 \%$ of $C$, dubliniensis were sensitive to Fluconazole, $93.75 \%$ of $C$. tropicalis, $88.8 \%$ of $C$. albicans, $75 \%$ of $C$ parapsilosis, $66.6 \%$ of C. guillermondi, 50 $\%$ of C. lusitanae, $40 \%$ of C. krusei, $20 \%$ of C. glabrata were sensitive to Fluconazole.

$100 \%$ of C. albicans and C. dubliniensis were sensitive to Voriconazole. $80 \%$ of $\mathrm{C}$. guillermondi and C. krusei, $75 \%$ of $C$. lusitanae, $60 \%$ of C. glabrata, $80 \%$ of $C$. parapsilosis and $95 \%$ of $C$. tropicalis were sensitive to Voriconazole

$6(4.6 \%)$ of the total isolates were resistant to both Fluconazole and Voriconazole. 4 out of 6 isolates belonged to $C$. glabrata and 2 belonged to $C$ tropicalis (Table 1-3).

\section{Zone diameter interpretive standards}

\begin{tabular}{|c|c|ccc|}
\hline Antifungal agent & Disk content & \multicolumn{3}{|c|}{ Zone Diameter, Nearest whole } \\
& & R & S-DD & S \\
\hline Fluconazole & $25 \mu \mathrm{g}$ & $\leq 14$ & $15-18$ & $\geq 19$ \\
\hline Voriconazole & $1 \mu \mathrm{g}$ & $\leq 13$ & $14-16$ & $\geq 17$ \\
\hline
\end{tabular}

Table.1 Antifungal susceptibility test

\begin{tabular}{|c|c|c|}
\hline Drug & Sensitive (\%) & Resistant (\%) \\
\hline Fluconazole & 65 & 35 \\
\hline Voriconazole & $\mathbf{8 2}$ & 18 \\
\hline
\end{tabular}

Table.2 Fluconazole susceptibility in Various Candida species isolated

\begin{tabular}{|c|c|c|}
\hline Candida species & Sensitive \% & Resistant \% \\
\hline C. albicans & 88.8 & 11.1 \\
\hline C. dubliniensis & 100 & 0 \\
\hline C. guillermondii & 66.6 & 33.3 \\
\hline C. krusei & 40 & 60 \\
\hline C. lusitanae & 50 & 50 \\
\hline C. parapsilosis & 75 & 25 \\
\hline C. glabrata & 20 & 80 \\
\hline C. tropicalis & 93.75 & 6.25 \\
\hline
\end{tabular}


Table.3 Voriconazole susceptibility in Various Candida species isolated

\begin{tabular}{|c|c|c|}
\hline Candida species & Sensitive $\%$ & Resistant $\%$ \\
\hline C. albicans & 100 & 0 \\
\hline C. dubliniensis & 100 & 0 \\
\hline C. guillermondii & 80 & 20 \\
\hline C. krusei & 80 & 20 \\
\hline C. lusitanae & 75 & 25 \\
\hline C. parapsilosis & 80 & 20 \\
\hline C. glabrata & 60 & 40 \\
\hline C. tropicalis & 95 & 5 \\
\hline
\end{tabular}

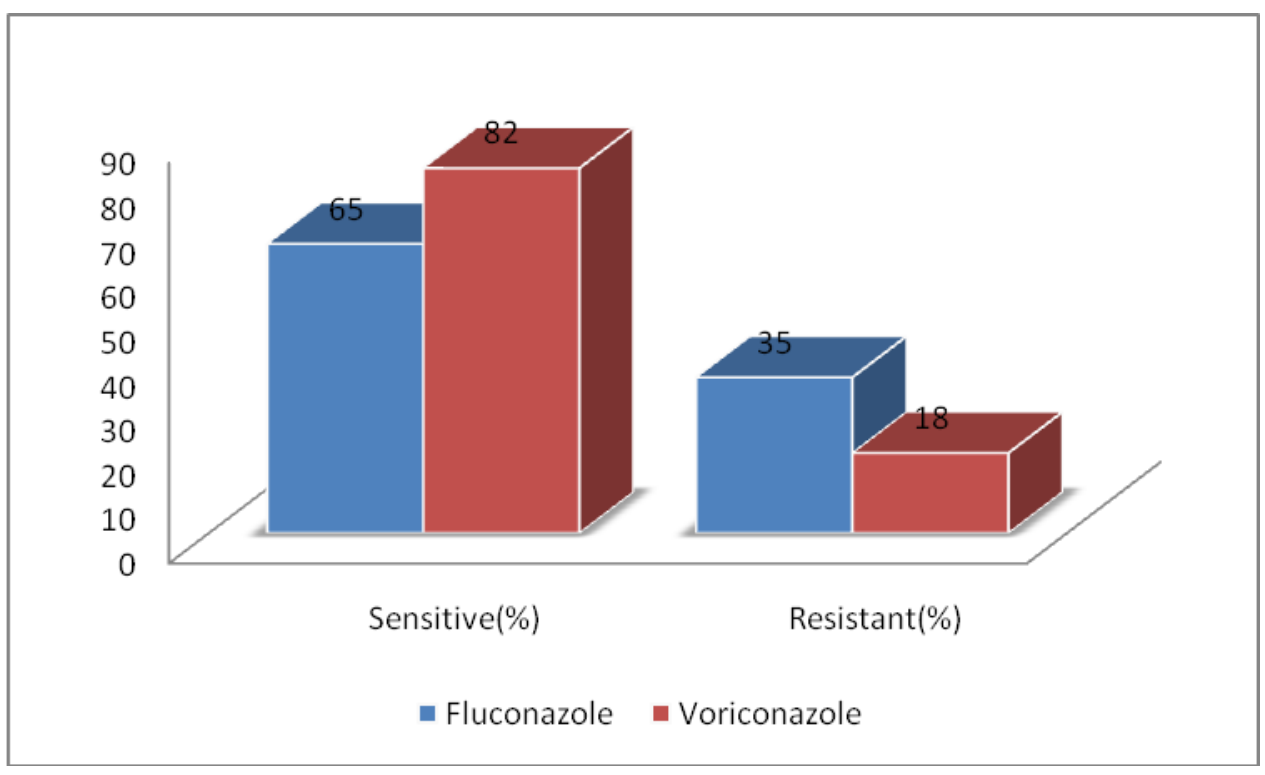

Antifungal Susceptibility Test

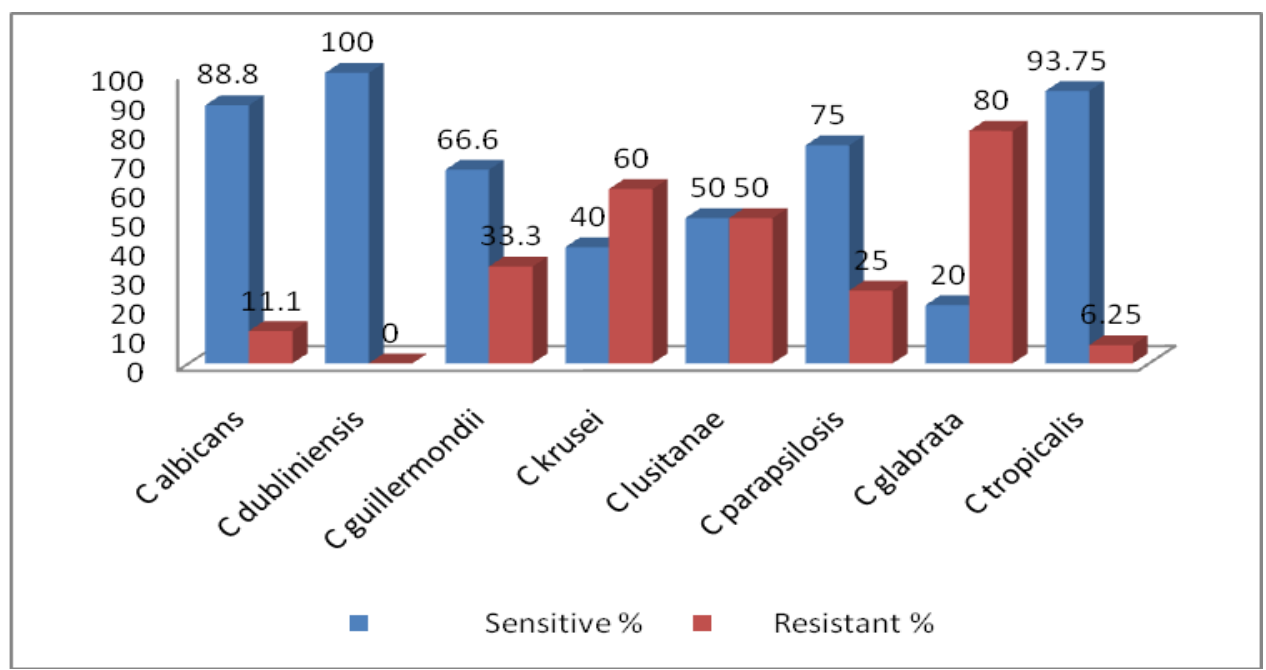




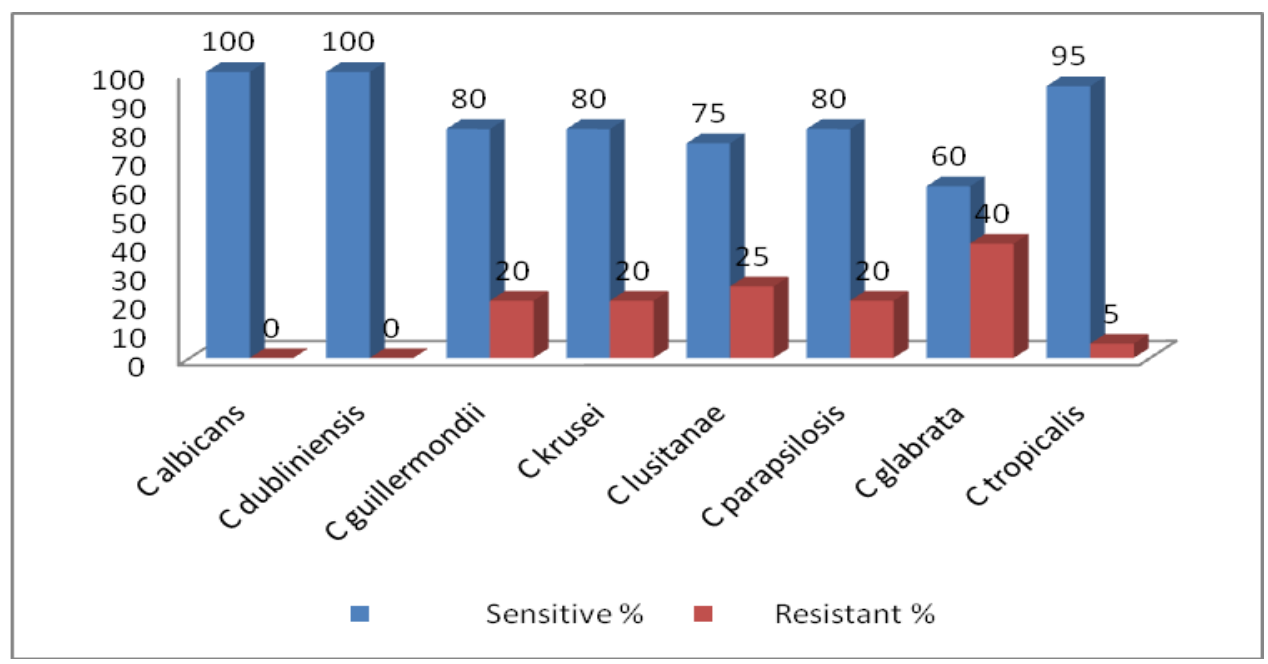

$6(4.6 \%)$ isolates of the total 130 isolates were resistant to fluconazole but sensitive to Voriconazole. Of which $C$. albicans, $C$. guillermondi (1each), $C$. glabrata and $C$. krusei (2 each). This was similar to a study conducted by Regha et al., which showed that $3.2 \%$ of total (95) isolates were fluconazole resistant but all were sensitive to voriconazole. $2.3 \%$ of $C$. tropicalis isolates and $9.5 \%$ of $C$. parapsilosis were fluconazole resistant but all were sensitive to voriconazole.

The triazoles remain active against many of isolates, Fluconazole is the first line antifungal agent for treating uncomplicated candidiasis (7-11). More fluconazole-resistant strains of endemic species may emerge whenever there are lapses in infection control precautions and extensive use of fluconazole. Voriconazole seemed to be superior to fluconazole with a better susceptibility in the fluconazole resistant strains.

\section{References}

1. Chander J. Candidiasis in Text book of medical mycology. $3^{\text {rd }}$ edition. : Mehta pub 2008: 267-290.

2. Chakrabarti A, Shivprakash M. Laboratory Diagnosis. In Medical Mycology Laboratory Procedures.2005:17-70.
3. M. A. Pfaller and D. J. Diekema Epidemiology of Invasive Candidiasis: a Persistent Public Health ProblemClin. Microbiol. Rev. 2007, 20(1):133

4. Richards M J, Edwards JR, Culver DH, Gaynes RP. Nosocomial infections in medical intensive care units in the United States. National Nosocomial Infections Surveillance System. Crit Care Med 1999;27: 887-892.

5. Ruan SY, Chen SY, Hsuek PR. Persistent Candida parapsilosis funguria associated with an indwelling urinary tract stent for more than 7 years. $J$ Med Microbiol 2008;5 :1585-7.

6. Sobel JD, Kauffman CA, McKinsey D, Zervos M, Vazquez JA, Karchmer AW et al. Candiduria: A randomized, doubleblind study of treatment with fluconazole and placebo. Clin Infect Dis 2000;30: 1924.

7. Larone D H. Medically Important Fungi; A guide to identification, Washington DC. ASM Press.2002; 328-329

8. W G Merz Candida albicans strain delineation. Clin. Microbiol. Rev. 1990, 3(4):321.

9. RJ Kothavade, MM Kura,AG Valand ,MH. Panthaki. Candida tropicalis: its prevalence, pathogenicity and increasing resistance to fluconazole. $\mathrm{J}$ Medl Microbiol (2010), 59, 873-880 
10. Kao AS, Brandt ME, Pruitt WR, Conn LA, Perkins BA, Stephens DS, et al. The epidemiology of Candidaemia in two United States cities: results of a population-based active surveillance. Clin Infect Dis 1999; 29 : 1164-70.
11. Verma AK, Prasad KN, Manisha S, Dixit AK, Ayyagari A. Candidaemia in patients of a tertiary health care hospital from north India. Ind J Med Res 2003;117 : 122-128.

\section{How to cite this article:}

Natasha, M.V. and Raksha, Y. 2019. Antifungal Susceptibility Profile of Candida and Its Comparison in Various Candida Species. Int.J.Curr.Microbiol.App.Sci. 8(06): 3022-3027. doi: https://doi.org/10.20546/ijcmas.2019.806.360 